\title{
AVALIAÇÃO DA PROPORCIONALIDADE DA MISTURA NA CINÉTICA DE CURA DA RESINA EPÓXI*
}

\author{
Maria Olivia de Barros Almeida ${ }^{1}$ \\ Michelli Siquiera Monteiro de Barros ${ }^{2}$ \\ Thamise Sampaio Vasconcelos Vilela ${ }^{3}$
}

\section{Resumo}

O intuito do presente trabalho é identificar, dentre as proporções entre resina e catalisador analisadas cineticamente, a melhor condição de cura. O mecanismo de cura das resinas epóxi é um dos mais estudados e a técnica mais utilizada no estudo deste comportamento é a calorimetria exploratória diferencial (DSC). Neste trabalho, foi utilizado o equipamento DSC 1, fabricada pela Mettler Toledo, pelo método dinâmico. Por meio da técnica de DSC, pode-se observar perfis de cura, temperatura de transição vítrea e a determinação das melhores condições de preparação de compósitos. A reação de cura de resinas termofixas é um processo complexo, definido como uma mudança irreversível nas propriedades químicas e físicas por uma reação química. Estabelecendo-se as condições ideais de cura, a resina epoxi resulta em uma estrutura rígida altamente reticulada, a qual combina as excelentes propriedades térmicas, mecânicas, resistência química.

Palavras-chave: Cura; Resina Epoxi; DSC.

\section{EVALUATIONS OF PROPORTION OF MIXTURE IN EPOXI RESIN CURE KINETICS}

\section{Abstract}

The present study objective is to identify the best cure condition between the resin and catalyst ratios kinetically analyzed. The curing mechanism of epoxy resins is one of the most studied and the technique used in the study this behavior is the Differential Scanning Calorimetry (DSC). For this study was DSC 1 equipment, by Mettler Toledo. The test method used is called, dynamic method. By DSC technique can be obtained the cure profiles, glass transition temperature and determination of the best conditions for preparing composites. The chemical reaction curing of thermosetting resins is a complex process, defined as an irreversible change in physical and chemical properties. Setting up the optimum conditions for curing, the epoxy resin results in a highly crosslinked rigid structure, which combines excellent thermal, mechanical, chemical resistance.

Keywords: Cure; Epoxy Resin; DSC.

1 Engenharia Química, Especialista de Desenvolvimento de Mateirais, Laboratório de Materiais, Prysmian Surflex Umbilicais e Tubos Flexíveis do Brasil Ltda, Cariacica, ES e Brasil.

2 Engenharia Química, Mestre, Especialista de Desenvolvimento de Mateirais, Laboratório de Materiais, Prysmian Surflex Umbilicais e Tubos Flexíveis do Brasil Ltda, Cariacica, ES e Brasil.

3 Engenharia Mecânica, Mestre, Especialista de Desenvolvimento de Mateirais, Laboratório de Materiais, Prysmian Surflex Umbilicais e Tubos Flexíveis do Brasil Ltda, Cariacica, ES e Brasil. 


\section{INTRODUÇÃO}

As resinas epóxi fazem parte de uma importante classe de polímeros termofixos, materiais estes que uma vez crosslincado tornam-se insolúveis e infusíveis. Atualmente estas resinas tem tido uma alta atenção em desenvolvimento.

Resina epoxi é definida como um polímero termofixo que enrijece quando se mistura um agente catalizador ou endurecedor [1], como por exemplo um diol (Bisfelnol A ou F) de alto peso molecular com epicloridrina.

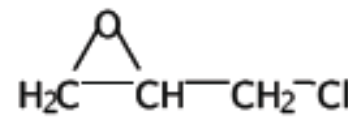

Epicloridrina

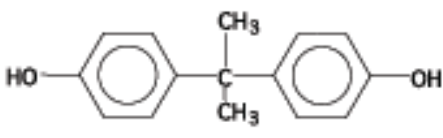

Bisfenol A

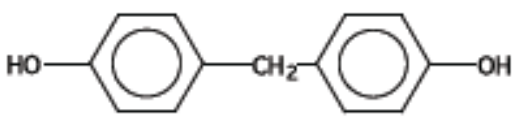

Bisfenol F

Figura 1. Materias utilizados para a produção de resina epoxi.

Comumente a epicloridrina e o Bisfenol A reagem conforme abaixo:

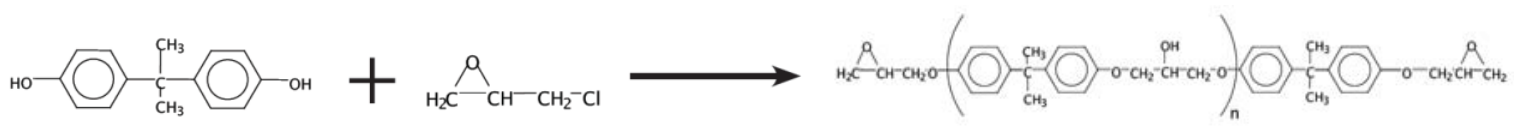

Figura 2. Reação química.

A reação de cura ou de reticulação de resinas termofixas é um processo complexo, definido como uma mudança irreversível nas propriedades químicas e físicas de uma determinada formulação resina/iniciador/promotor causada por uma reação química. A reação de reticulação da resina é altamente exotérmica. Tal reação pode ser facilmente controlada ajustando o ambiente de cura, que é realizado conhecendo-se a transferência de calor que ocorre durante o processo e a cinética de cura. A seleção das condições de cura é fundamental, uma vez que afetam o comportamento mecânico da resina reticulada. Estabelecendo-se as condições ideais de cura, a resina resulta em uma estrutura rígida altamente reticulada, a qual combina as excelentes propriedades térmicas, mecânicas, resistência química e à solventes [2;3]. Além das condições de cura, a presença de cargas ou reforços também pode influenciar o comportamento de cura dessas resinas, sugerindo que as propriedades interfaciais são afetadas por interações carga-resina [3]. Por esta razão, é muito empregada na confecção de piscinas, tanques de armazenamento de solventes, tubulações de esgoto, entre outros. As resinas são utilizadas em diversos setores e um deles é na ancoragem da armadura de tração encontrada no conector end fitting, utilizadas em tubos flexíveis. É dentro destes componentes que os arames da armaduras de tração são fixados por resina epóxi em conjunto com uma configuração específica permitindo dessa maneira a sustenção do duto flexível quando fixado a plataformas offshore [4], figura 3. 

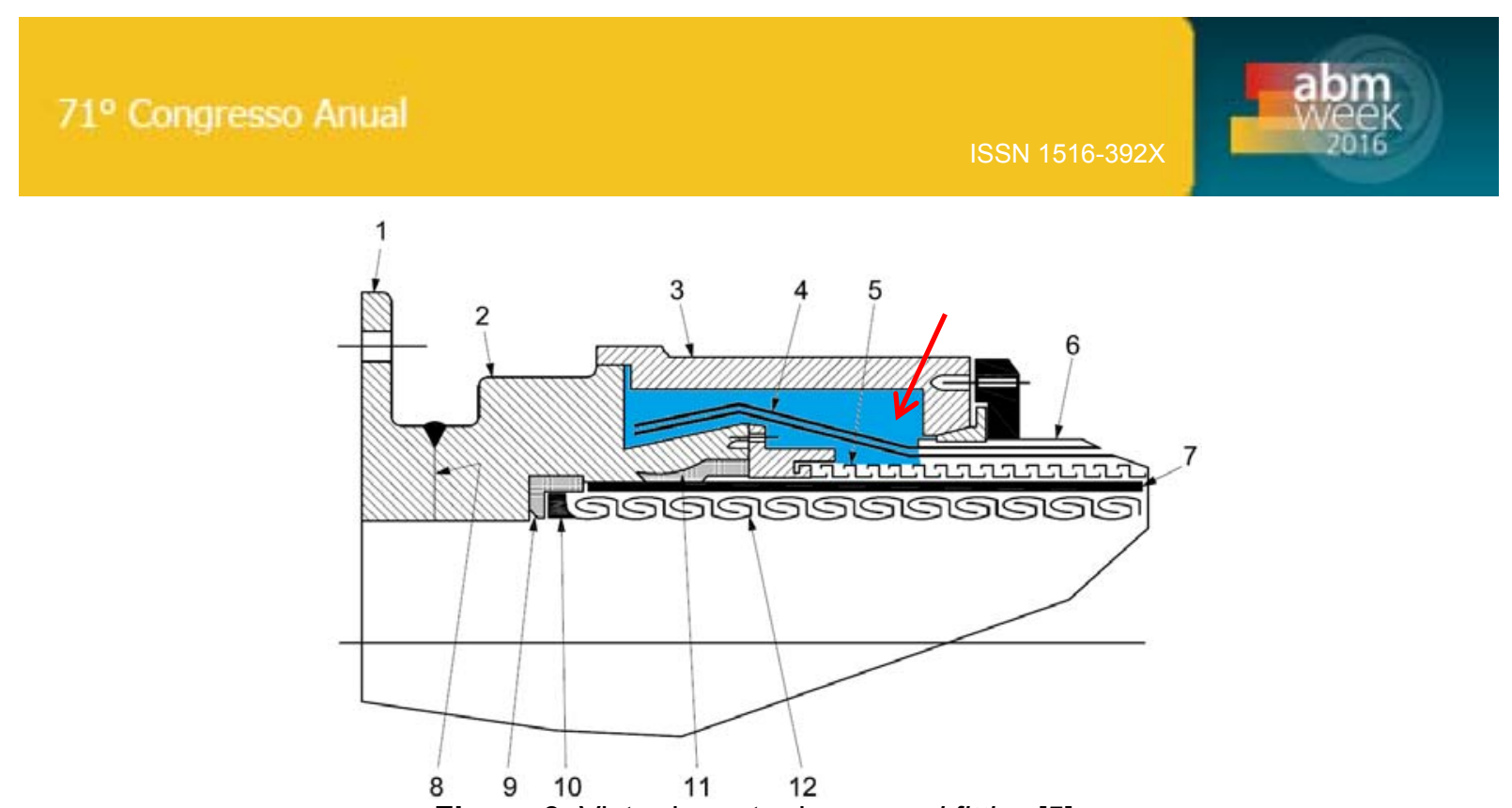

Figura 3. Vista de corte de um end fitting [5].

Segundo a API 17B [5], as duas finalidades propostas para os end fitting são: finalizar o tubo flexível de modo que as cargas axiais e o momento de flexão possa ser transmitido para o conector sem afetar as camandas onde se encontram os fluidos e fornecer uma resistência a pressão durante a transição entre o tubo flexível e o conector.

A calorimetria diferencial exploratória (DSC) tem sido usada com sucesso no estudo da reação de cura de resinas epóxi visando estimar parâmetros cinéticos. Os métodos dinâmicos tais como: o método de Borchardt e Daniels, de Kissinger, de Ozawa e o método Barrett, oferecem resultados mais rápidos quando comparados aos métodos isotérmicos. A maioria dos estudos onde se utiliza a técnica de DSC no monitoramento das reações de cura de resinas epóxi comerciais refere-se a compostos bi, tri e multifuncionais [6].

A análise térmica é uma técnica que baseia a medida de uma propriedade em função da temperatura por um determinado tempo.

O objetivo deste trabalho é avaliar através da cinética de cura pelo método dinâmico a melhor proporção de cura para o material analisado.

\section{MATERIAIS E MÉTODOS}

As resinas analisadas foram a Rengel SW404 e endurecedor REN HY 404 BR, do fornecedor Hustman. Para o presente trabalho foram analisados 3 proporções de resina/endurecedor $(8: 1 ; 10: 1$ e 12:1). As amostras preparadas foram aquecidas em 3 velocidades diferentes $05^{\circ} \mathrm{C} / \mathrm{min}$; $10^{\circ} \mathrm{C} / \mathrm{min}$ e $20^{\circ} \mathrm{C} / \mathrm{min}$. Pesou-se aproximadamente $10 \mathrm{mg}$ das misturas não-curadas em porta-amostras de alumínio fechadas de $40 \mu \mathrm{l}$ e foram submetidas as razões de aquecimentos já informada, na faixa de temperatura de $-40^{\circ} \mathrm{C}$ a $200^{\circ} \mathrm{C}$, em um DSC Mettler Toledo (DSC 1). Com resfriamento Hubber.

Os parâmetros apresentados neste trabalho foram calculados através do programa utilizado pelo equipamento.

\section{RESULTADOS E DISCUSSÃO}

Através das corridas nos ensaios de cinética realizados apresentados nas figuras 4 a 6 foi possível realizar o cálculo da energia de ativação das proporções das misturas analizadas, conforme demostrado na tabela 1. 


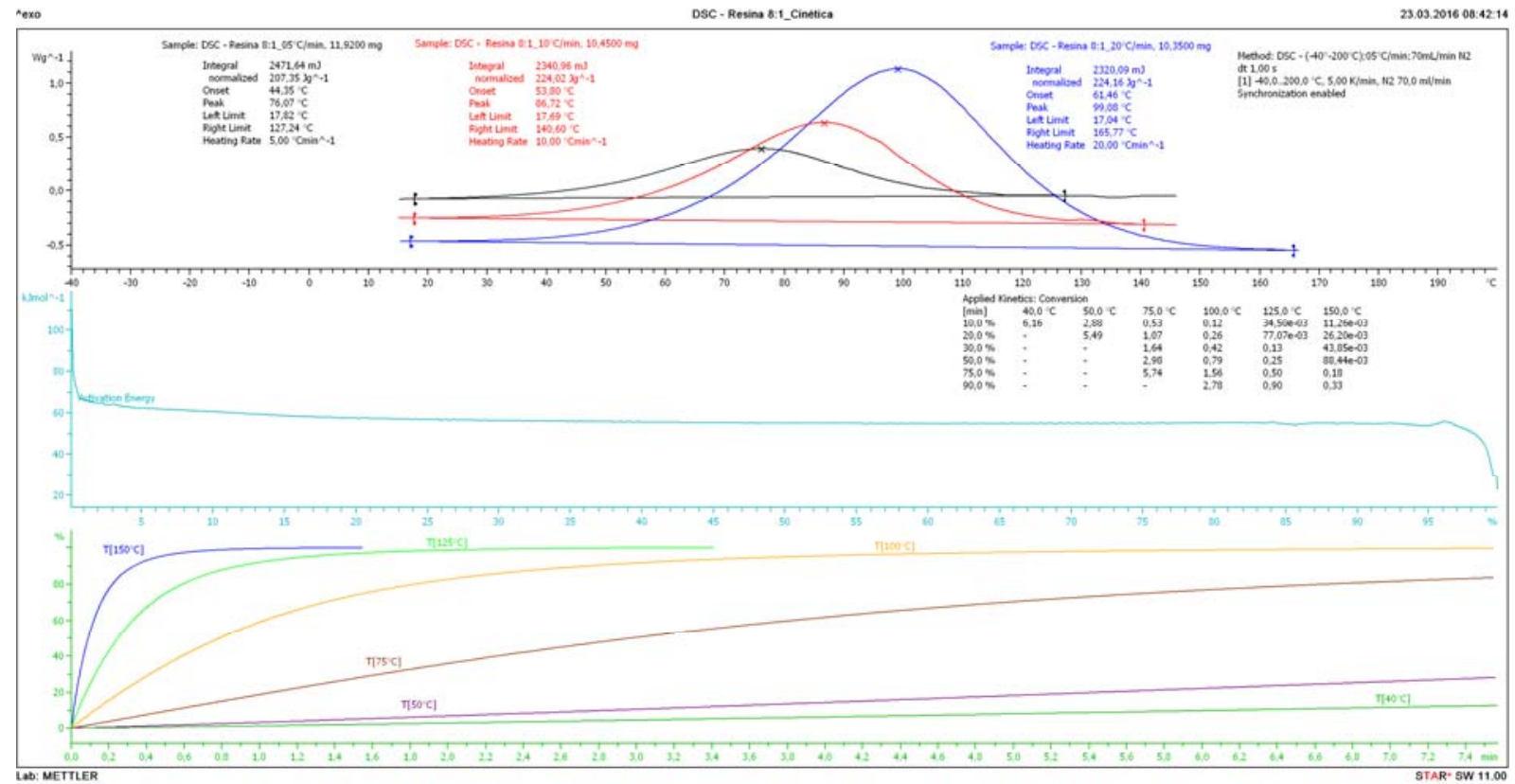

Figura 4. Análise de Cinética da proporção 8:1.

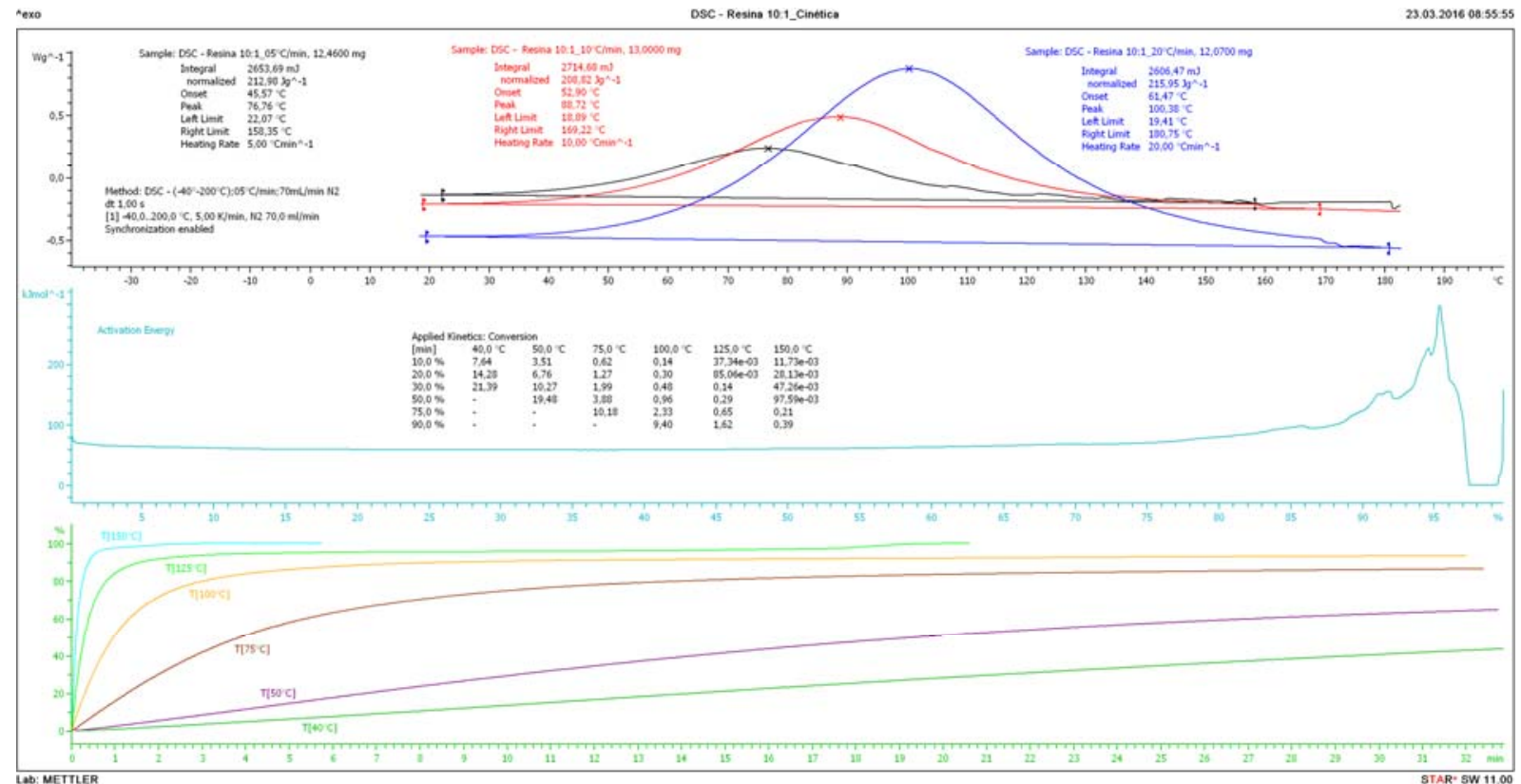

Figura 5. Análise de Cinética da proporção 10:1. 


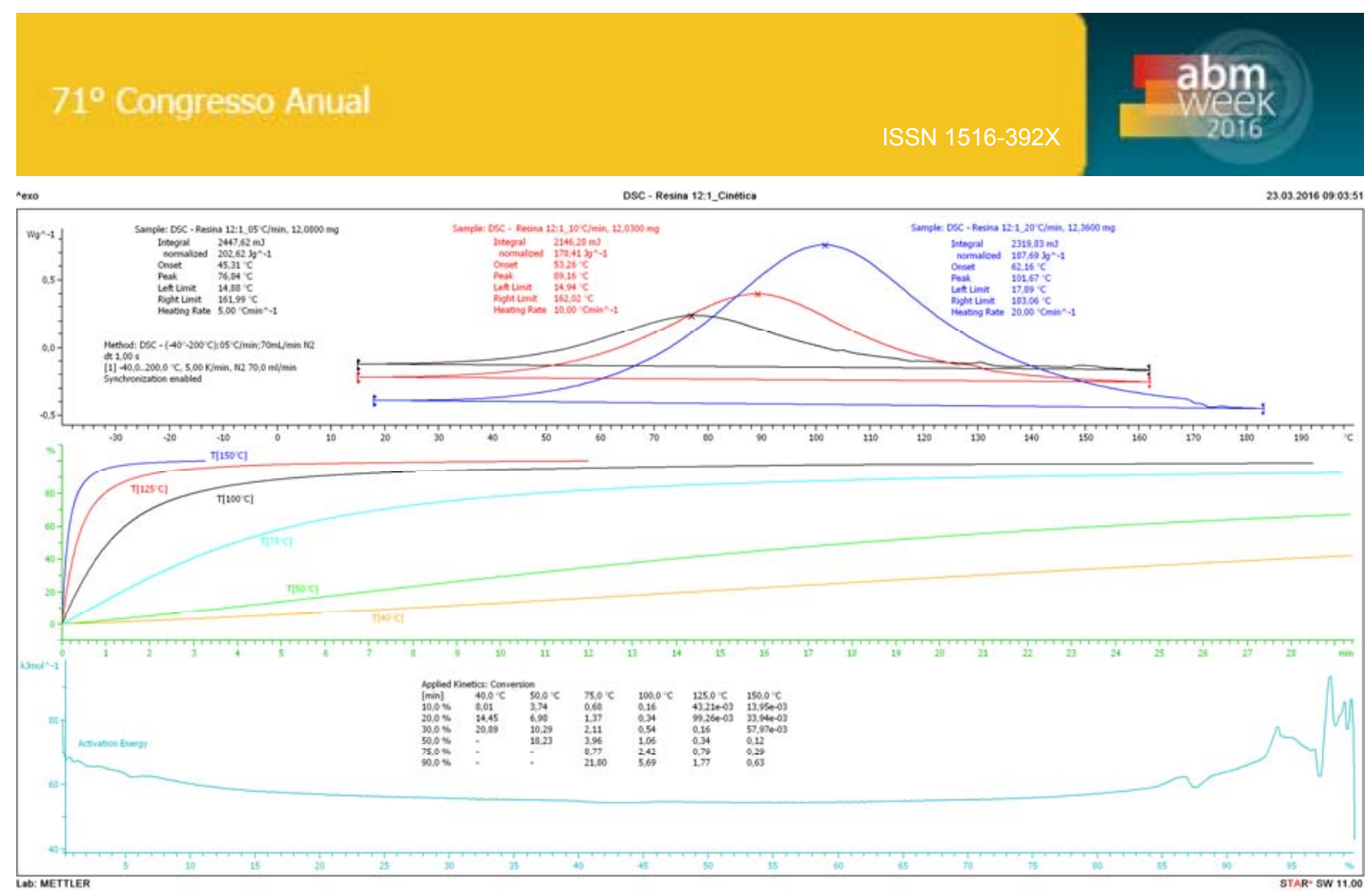

Figura 6. Análise de Cinética da proporção 12:1.

Por meio dos ensaios realizado e com base na ASTM E 698[7] é possível a elaboração do gráfico abaixo (figura 7) obtendo a curva para o cálculo da energia de ativação.

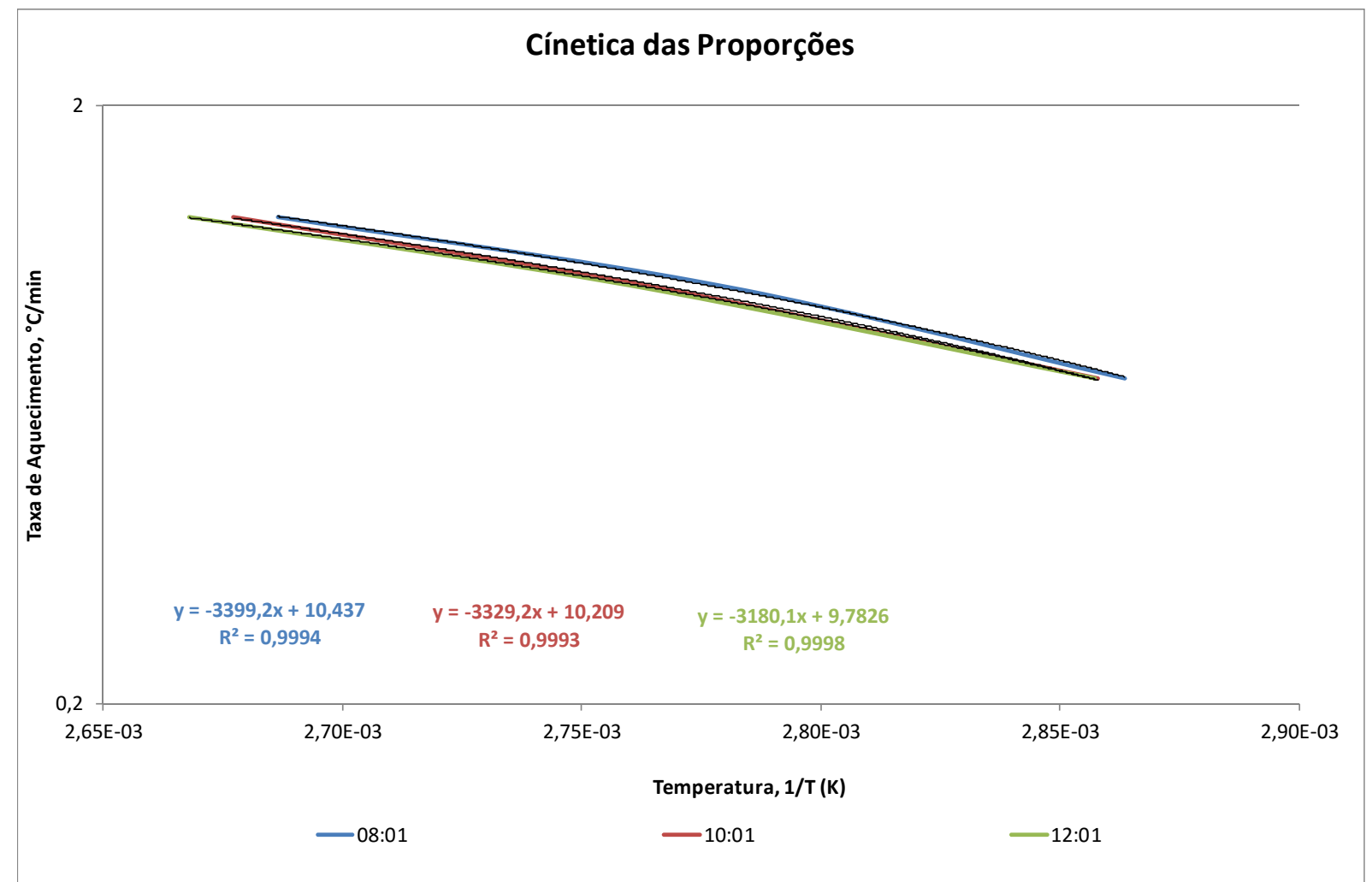

Figura 7. Curva de Cinética.

Através dos resultados obtidos é possível verificar que a energia de ativação necessária para o início da reação de cura descresce a medida que aumentamos a parte da resina nas proporções avaliadas, isto acontece pois, a fração do endurecedor diminiu. 
Tabela 1. Tabela de Energia de Ativação

\begin{tabular}{|c|c|c|c|c|c|c|}
\hline \multicolumn{7}{|c|}{ Energia de Ativação } \\
\hline \multirow{2}{*}{ Proporção } & \multirow{2}{*}{$\begin{array}{c}\begin{array}{c}\text { Taxa de } \\
\text { Aquecimento }\end{array} \\
{ }^{\circ} \mathrm{C} / \mathrm{min}\end{array}$} & \multicolumn{3}{|c|}{ Temperatura } & \multirow{2}{*}{$\begin{array}{l}\Delta \mathrm{H} \\
\mathrm{J} / \mathrm{g}\end{array}$} & \multirow{2}{*}{$\begin{array}{c}\mathrm{E}^{\prime \prime} \\
\mathrm{kJ} / \mathrm{mol}\end{array}$} \\
\hline & & ${ }^{\circ} \mathrm{C}$ & $\mathbf{K}$ & $1 / K$ & & \\
\hline \multirow{3}{*}{$8: 1$} & 5 & 76,07 & 349,22 & 0,002864 & 207,35 & 59427,47 \\
\hline & 10 & 86,72 & 359,87 & 0,002779 & 224,02 & 59168,15 \\
\hline & 20 & 99,08 & 372,23 & 0,002687 & 224,16 & 59168,15 \\
\hline \multirow{3}{*}{$10: 1$} & 5 & 76,76 & 349,91 & 0,002858 & 212,98 & 58203,68 \\
\hline & 10 & 88,72 & 361,87 & 0,002763 & 208,82 & 57949,70 \\
\hline & 20 & 100,38 & 373,53 & 0,002677 & 215,95 & 57671,82 \\
\hline \multirow{3}{*}{$12: 1$} & 5 & 76,84 & 349,99 & 0,002857 & 202,62 & 55354,39 \\
\hline & 10 & 89,16 & 362,31 & 0,00276 & 178,41 & 55088,96 \\
\hline & 20 & 101,67 & 374,82 & 0,002668 & 187,69 & 54801,39 \\
\hline
\end{tabular}

Realizando um comparativo das curvas de conversões dos dados obtidos pela curva de DSC entre as proporções nota-se uma ligeira superioridade para a proproção 8:1, ou seja precisa de menos tempo para atingir o cura de $100 \%$. Isto pode ser verificado através das figuras 8 a 10.

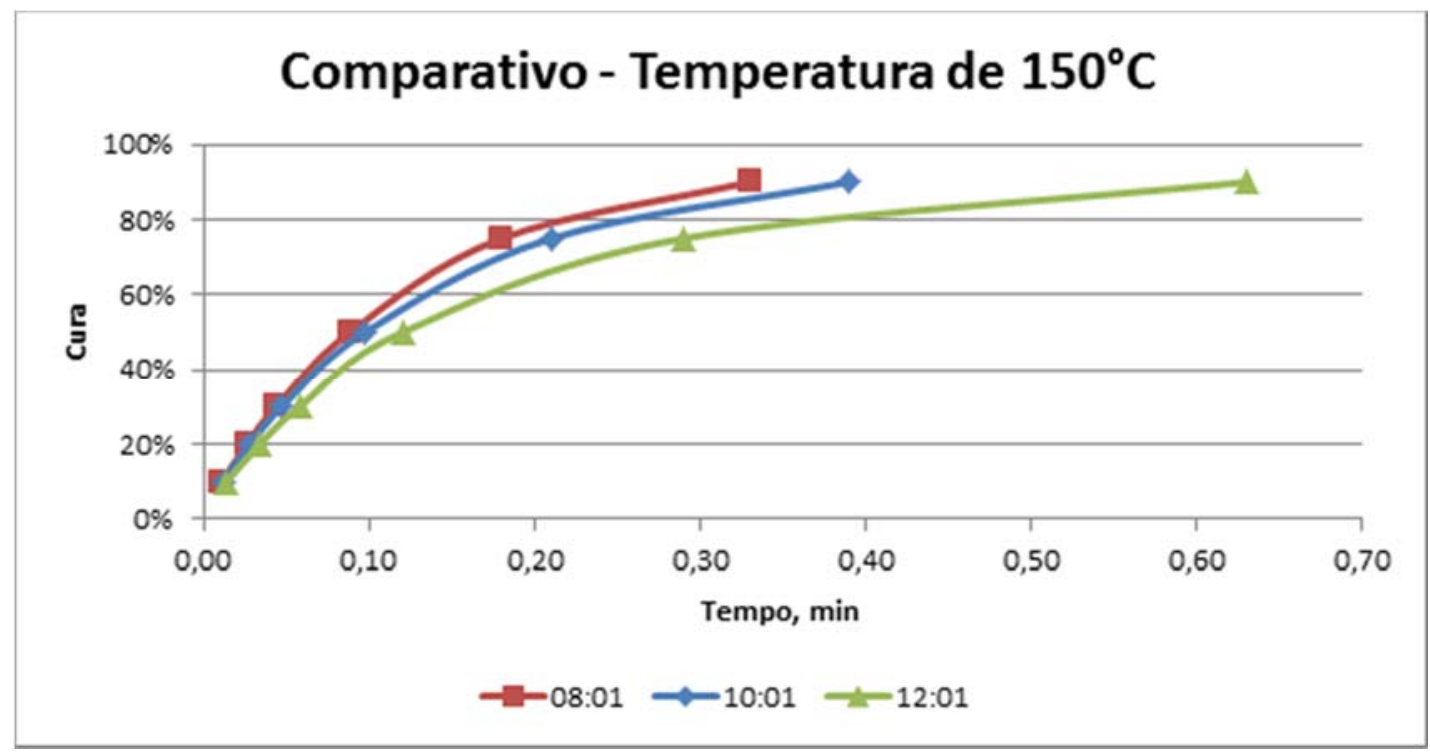

Figura 8. Comparativo $-150^{\circ} \mathrm{C}$. 


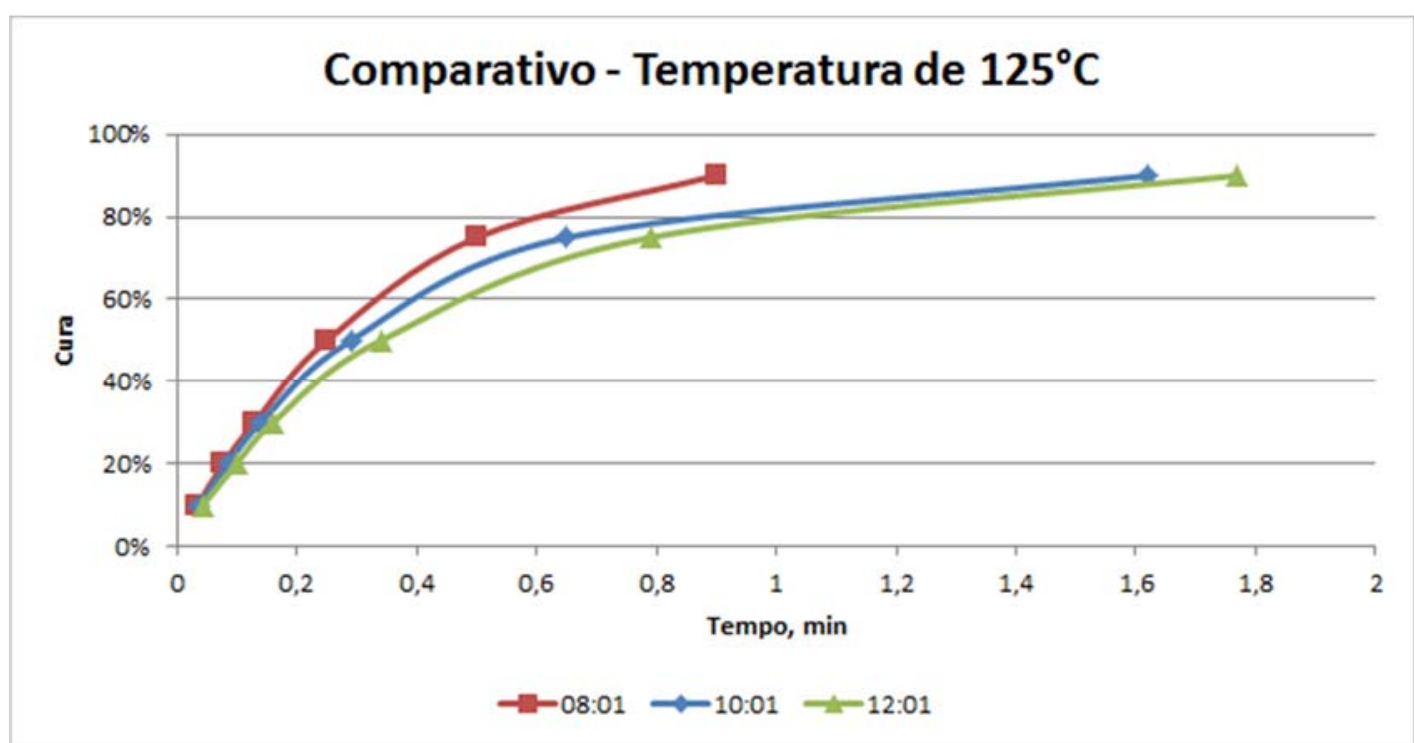

Figura 9. Comparativo $-125^{\circ} \mathrm{C}$.

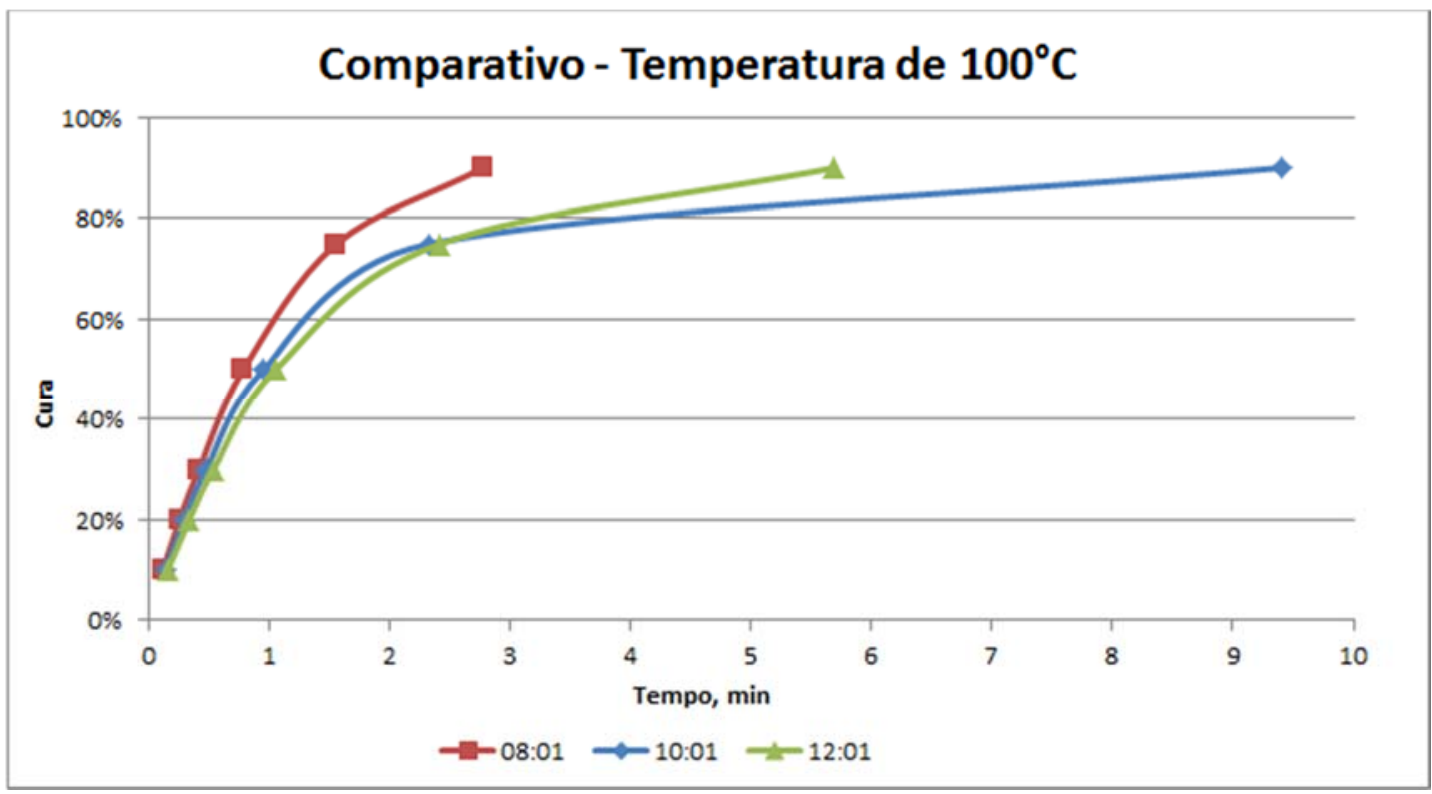

Figura 10. Comparativo $-100^{\circ} \mathrm{C}$.

\section{CONCLUSÃO}

A caracterização por DSC mostrou que o uso de diferentes proporções da resina epoxi/endurecedor resultou na diminuição dos valores obtidos de energia de ativação. Isto ocorreu devido ao redução do endurecedor nas razões de misturas analisadas.

Para as mesmas velocidades de aquecimento a temperatura do pico de reação aumenta para as diferentes razões analisadas.

A temperatura de cura da mistura aumenta com o aumento da resina na proporção. A análise de cinética apresentou resultado ligeiramente superior para a proporção de 8:1. Através das análises realizadas, pode-se concluir que a proporção 8:1 atingirá primeiro a cura.

Para trabalhos futuros propomos utilizar o método isotérmico e avaliar melhor as proporções citadas nestes trabalho. 


\section{Agradecimentos}

Agradecemos a empresa Prysmian Group - Surflex por proporcionar a oportunidade de execução deste trabalho.

\section{REFERÊNCIAS}

1 Definição de resina epoxi, disponível em https://pt.wikipedia.org/wiki/Ep\%C3\%B3xi, [acesso em 14/03/2016].

2 Rodrigues, MRA; Estudo da Reação de Cura da Resina Epoxi (Araldit F) com Anidrido Ftálico e Trietilamina como Indicador, Dissertação de Mestrado, 1991, Universidade Federal do Rio Grande do Sul.

3 Ittner CA e Felisberti MI. Cinética de Cura de Compósito à Base de Resina Éster Vinílica e Sílica Funcionalizada. CBECIMAT. 2000 [acesso em 07 mar. 2016]; Disponível em: http://gppol.iqm.unicamp.br/Congressos/14CBECIMAT/TC403-009.pdf.

4 Xavier FG. Avaliação da Vida em Fadiga de um Novo Modelo De Terminal Conector para Dutos Flexíveis. Dissertação de Doutorado. 2009, Universidade Federal do Rio Grande do Sul.

$5 \quad$ API 17B, Recomended Practice for Flexible Pipe, API, 2014.

6 Costa APO, Gerbase AE, Petzhold CL. Investigação da Cinética de Cura por Calorimetria Diferencial Exploratória (DSC) de Resinas Epóxi Preparadas a partir de Óleo de Soja Epoxidado com Diferentes Anidridos e Aminas Terciárias. Revista Polímeros vol $21 \mathrm{n}^{\circ}$ 2. 2011: 146-150.

7 ASTM E 698 - 11. Standard Test Method for Arrhenius Kinetic Constants for Thermally Unstable Materials Using Differential Scanning Calorimetry and the Flynn/Wall/Ozawa Method. 